\title{
From systematic lymphadenectomy to sentinel lymph node mapping: a review on transitions and current practices in endometrial cancer staging
}

\author{
Chantal A. L. Stämpfli ${ }^{1}$, Andrea Papadia ${ }^{2}$, Michael D. Mueller ${ }^{1}$ \\ ${ }^{1}$ Department of Obstetrics and Gynecology, University Hospital of Bern and University of Bern, Bern, Switzerland; ${ }^{2}$ Department of Obstetrics and \\ Gynecology, Ente Ospedaliere Cantonale, University of the Italian Switzerland, Lugano, Switzerland \\ Contributions: (I) Conception and design: CAL Stämpfli; (II) Administrative support: None; (III) Provision of study materials or patients: None; (IV) \\ Collection and assembly of data: None; (V) Data analysis and interpretation: CAL Stämpfli, A Papadia; (VI) Manuscript writing: All authors; (VII) \\ Final approval of manuscript: All authors. \\ Correspondence to: Prof. Dr. med. Michael D. Mueller. Department of Obstetrics and Gynecology, University Hospital of Bern and University of Bern, \\ Bern, Switzerland. Email: michel.mueller@insel.ch.
}

\begin{abstract}
Endometrial cancer care has undergone major changes in the past 30 years. In 1988, staging transitioned from clinical to surgical. Moreover, the surgical approach of choice is no longer open surgery, but minimally invasive surgery. An improvement in terms of nodal evaluation followed. Full systematic lymphadenectomy has been continuously replaced by sentinel lymph node mapping. Although sentinel lymph node mapping with a cervical injection of indocyanine green dye is rapidly gaining clinical acceptance, we lack consistent recommendations on a well-defined procedure that accurately and indolently assesses the lymph node status. Such recommendations are indispensable, as nodal status is the most important predictive factor of survival and is essential for tailoring adjuvant treatment to the risk of recurrence. This paper focuses on transitions in endometrial cancer care and highlights current data on sentinel lymph node mapping in endometrial cancer. We demonstrate that sentinel lymph node mapping is a safe and accurate strategy for nodal status evaluation with appropriate sensitivity, false-negative rate and negative predictive value in high- as well as low-risk settings. Furthermore, we elaborate on type and dose of tracer, site of injection, number of sentinel lymph nodes to be removed, sentinel lymph node mapping learning curve, operation mode and sentinel lymph node ultrastaging. In the future, guidelines with consistent recommendations on the above outlined features of sentinel lymph node mapping should be established to allow for a uniform and wide-spread application of the sentinel lymph node mapping procedure.
\end{abstract}

Keywords: Endometrial cancer (EC); sentinel lymph node mapping; indocyanine green; minimally invasive surgery; ultrastaging; gynecologic oncology

Submitted Oct 08, 2020. Accepted for publication Jan 27, 2021.

doi: $10.21037 / \mathrm{cco}-20-224$

View this article at: http://dx.doi.org/10.21037/cco-20-224

\section{Introduction: transitions in endometrial cancer staging}

Endometrial cancer (EC) are a diverse group of neoplasms with a varying degree of aggressiveness, especially in early stages. An appreciable number of patients (14-22\%) with stage I cancers have disease outside of the uterus (lymph node metastasis, adnexal disease, intraperitoneal spread and/ or malignant cells in peritoneal washings), thereof, $11 \%$ had pelvic (9\%) and/or paraaortic (6\%) nodal metastases (1).

Nodal status is the strongest predictive factor of survival and is essential for tailoring adjuvant treatment to the risk of recurrence. As nodal status and therefore true extent of disease can only be reliably assessed by surgical staging, the International Federation of Gynecology and 
Obstetrics (FIGO) transitioned the EC staging from clinical to surgical in 1988 (2). The conventional surgical staging for endometrial cancer includes visual evaluation of the peritoneal cavity, total hysterectomy, bilateral salpingo-oophorectomy and a pelvic and paraaortic lymphadenectomy.

However, the performance of a pelvic and paraaortic lymphadenectomy for nodal status evaluation has been faced with controversy ever since its implementation. The procedure has been conducted reluctantly and with low compliance $(35 \%, 66 \%$ and $90 \%$ performance in grade 1, 2 and 3 tumors, respectively) (3). However, by leaving nodal status unknown, incomplete resection of disease and prescription of inadequate adjuvant treatment is risked. As the true risk for nodal involvement is assessed by postoperative permanent pathological examination of the uterine specimen, presumed low-risk EC patients may end up having a high-intermediate- or high-risk EC with an up to $40 \%$ risk of nodal metastases. As a result, highintermediate-risk EC patients with unknown nodal status have a worse prognosis than women with known nodal status, irrespective of whether or not the lymph nodes are metastatic. To be precise, 5 -year survival rates are $85 \%$, $71.8 \%$ and $36 \%$ when nodal status is negative, positive or unknown, respectively (4).

Several reasons contribute to the restraint of conducting a full systematic lymphadenectomy in every EC patient. First and foremost, the surgical morbidity of a full systematic lymphadenectomy is high. This is relevant, as the majority of EC patients are co-morbid and do not suffer from metastatic disease. Secondly, lymphadenectomy is technically difficult to master in obese women, representing a large proportion of type I EC patients. Thirdly, the procedure was originally adopted despite the lack of evidence demonstrating improved survival. Last but not least, the therapeutic value of lymphadenectomy is still questioned (5-7).

Over the past years, less invasive and more tailored strategies for nodal evaluation have been investigated. These include selective lymph node sampling (caveat: only $10 \%$ metastatic nodes present grossly enlarged) (1), triaging patients to lymphadenectomy based on intrauterine risk factors at frozen section of the uterus (caveat: accurate risk stratification can only be made postoperatively after permanent pathological examination of the uterine specimen) $(4,8)$ and triaging patients to lymphadenectomy based on nodal status attained by sentinel lymph node (SLN) mapping. Best results are achieved when SLN mapping is applied (i.e., sensitivity $97.2 \%$, false-negative rate $2.8 \%$, negative predictive value 99.6\%) (9).

SLN mapping in EC was first described in 1996 (10). With increasing acceptance in clinical practice, it is just now progressively being incorporated in international guidelines (11-13). However, there is still concern about the safety of SLN mapping in high-risk settings. In high-risk EC, the probability of nodal metastasis (and therefore occult stage IIIC disease) is high and underdiagnosis is risked if the procedure does not achieve a low false-negative (FN) rate (14). To improve the FN rate, Barlin et al. (2012) proposed the MSKCC (Memorial Sloan Kettering Cancer Center) SLN mapping algorithm. It goes beyond the sole removal of the mapped SLNs. Performance of ultrastaging is advised if the initial conventional histological examination of the lymph node is negative. Furthermore, removal of suspicious non-SLNs and performance of a side-specific pelvic lymphadenectomy are recommended in case of mapping failure on one hemipelvis. Paraaortic lymphadenectomy is left at the physician's discretion. Thereby, FN rate can be decreased from $15 \%$ to under $2 \%$, a value similar to the one seen in the treatment of other malignancies (15).

In the following paragraphs, we will review current evidence on surgical staging and SLN mapping in EC, highlighting standards and research on the operation mode, type and dose of tracer, site of injection, number of SLNs to be removed, SLN mapping learning curve, and SLN ultrastaging. Moreover, clinical applicability and oncological outcome of SLN mapping in EC will be reviewed.

\section{Surgical staging und SLN mapping in EC: non- uniformly handled features}

\section{Operation mode: minimally invasive surgery versus open surgery}

In the past, EC staging has been performed through laparotomy. However, there has been a shift towards minimally invasive surgery (MIS) in the past decades. The LAP2 trial, a prospective randomized trial involving early-stage EC patients demonstrated that laparoscopic EC staging has multiple advantages over the laparotomy approach. A decrease in postoperative adverse events and duration of hospital stay were noted. Intraoperative injuries were not increased despite the longer operation duration. Detection of advanced disease was the same in both groups, which means that MIS does not compromise surgical staging (16). The LACE trial, a multinational randomized 


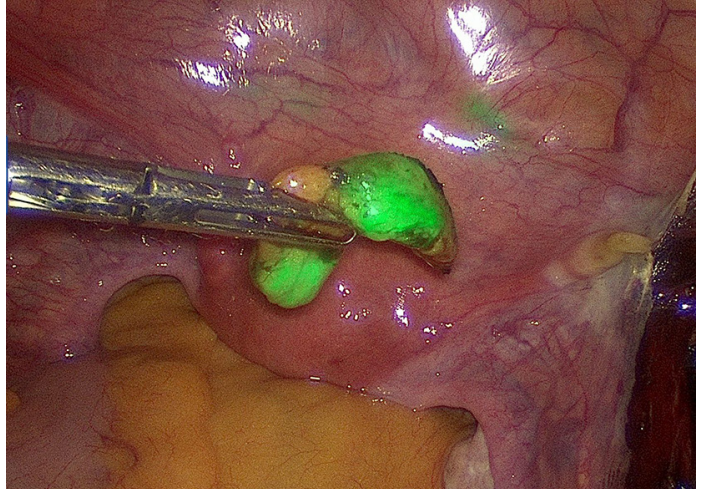

Figure 1 SLN mapped with ICG tracer and visualized with IMAGE1 STM RUBINA ${ }^{\mathrm{TM}}$ system.

equivalence trial involving early-stage EC patients also supported the safety of a laparoscopic approach. Diseasefree and overall survival after a follow-up of 4.5 years were comparable in the laparoscopy and laparotomy group (17). As nodal spread of disease was relatively low in the two just mentioned study populations, the safety of MIS in stage IIIC patients could not be assessed. In stages with nodal involvement, complete resection of disease is prognostically highly relevant. Papadia et al. (2020) compared the laparoscopic approach to laparotomy in stage IIIC patients. They reported that complete resection of disease is accomplished with equal success irrespective of surgical approach. Both groups had a similar 5-year overall survival rate. At multivariate analysis, only age over 65 years was associated with impaired overall survival. Due to the complexity of the surgery, operative time was longer in the laparoscopy group (325 vs. $264 \mathrm{~min}$ ). Despite the longer operative time in this setting, perioperative complications, estimated blood loss $(890$ vs. $380 \mathrm{~mL})$ and need for transfusion $(67 \%$ vs. $20 \%$ ) were significantly lower in the laparoscopic group. Thus, laparoscopic surgery is a safe and feasible surgical staging strategy in stage IIIC disease (18). These results are consistent with other studies' findings (19-21). Moreover, sensitivity, FN rate and negative predictive value (NPV) are not impaired by MIS $(9,22)$. Gehrig et al. (2008) investigated the optimal MIS strategy for comprehensive staging in obese and morbidly obese women, constituting the majority of EC patients. They concluded that roboticassisted surgery leads to shorter operative time, less blood loss, and shorter hospital stay (23).

Nowadays, the German S3 guidelines support the use of
MIS in presumed early-stage disease (11). According to the US NCCN guidelines, MIS is the preferred approach when technically feasible (12). The European ESMO-ESGOESTRO guidelines recommend MIS for the surgical management of low- and intermediate-risk EC. It can be considered in high-risk EC as well (13).

\section{Type of tracer}

Tc-99m, blue dyes (i.e., methylene blue, isosulfan blue and patent blue) and indocyanine green (ICG) are the most commonly used tracers for SLN mapping in EC. They can be detected by radionuclide, colorimetric or near-infrared methods, respectively. The effectiveness of these tracers, either alone or in combination, is indicated by their overall and bilateral detection rates. Overall detection describes the mapping of at least one SLN in either hemipelvis, whereas bilateral detection describes the mapping of at least one SLN in each hemipelvis. Only if mapping occurs in both hemipelvises, a side-specific pelvic lymphadenectomy can safely be omitted (i.e., SLN mapping is accurate on its own).

Bilateral and/or overall detection rates are highest for near-infrared ICG SLN mapping. SLN mapping with blue dye alone or in combination with Tc-99m is less accurate. To be concrete, overall detection rates range from $87-100 \%$ for ICG, $95-97 \%$ for a combination of blue dye with Tc- $-99 \mathrm{~m}$ and $57-81 \%$ for blue dye alone. Bilateral detection rates range from $65-88 \%$ for ICG, $50-74 \%$ for blue dye in combination with Tc-99m and $32-54 \%$ for blue dye alone (24-31). These findings have been confirmed in a recent randomized phase 3 trial (32).

Gasparri et al. suggest that the high detection rates achieved by using ICG result from the long retention of the tracer in the SLNs. This might contribute to a less timepressured SLN sampling, resulting in higher detection rates. However, special attention has to be drawn not to mistake echelon lymph nodes for SLNs, as sampling of those would increase surgical morbidity without increasing FN rate (33). Further benefits of ICG include its excellent toxicity profile (severe allergic reactions $<0.05 \%$ ), low cost and quick transcutaneous real-time visualization. Moreover, it attenuates the detrimental effect of body mass index (BMI) on detection rates (34). Based on this evidence, ICG is currently the preferred tracer for SLN mapping in EC. For illustration see Figure 1: SLN mapped with ICG tracer and visualized with IMAGE1 $\mathrm{S}^{\mathrm{TM}}$ RUBINA ${ }^{\mathrm{TM}}$ system (Karl Storz). 


\section{Dose of tracer}

As ICG is only FDA-approved for intravenous injections, its dose for interstitial injections has been set empirically. A metaanalysis on detection rate and diagnostic performance of ICG suggests that ICG achieves best performance when applied in large volumes and low concentrations ( 0.5 to $1.25 \mathrm{mg} / \mathrm{mL}$ ) (35). Most centers follow the dosage used by Frumovitz et al. (2018) at the FILM-trial: $1 \mathrm{~mL}$ of a $1.25 \mathrm{mg} / \mathrm{mL}$ ICG solution per superficial and deep injection (32).

Papadia et al. (2018) investigated the impact of different doses of ICG on detection rate. Although they were not able to show any correlation of dose to detection rate, they pointed out that the number of SLNs removed correlates with volume and concentration of ICG. It is however unclear whether the additionally sampled lymph nodes were true SLNs of independent lymphatic pathways or echelon lymph nodes (36).

\section{Site of injection}

As the uterus is a midline structure, its lymphatic drainage is complex. Four different uterine lymphatic draining systems are described in literature. Most metastatic spread happens along the lymph nodes of the upper paracervical pathway (37). As a theoretical difference between the lymphatic drainage of the cervix and the uterine corpus must be expected, there is no consensus on the optimal site of tracer injection. Three different sites of injection exist: cervical injection, hysteroscopic myometrial/peritumoral injection and transabdominal subserosal/myometrial injection at the uterine fundus.

The cervical injection route is the most practical and shows the highest technical success rate. Specifically, the ICG solution is injected superficially into the submucosa $(2-3 \mathrm{~mm})$ and deeply into the stroma $(1-2 \mathrm{~cm})$ of the four cervical quadrants (38). Overall and bilateral detection rates are $62-100 \%$ and $57-98 \%$, respectively $(37,39,40)$. A hysteroscopic injection of tracer does not only result in lower detection rates (e.g., due to intraabdominal leakage of ICG and a longer learning curve) but is also more challenging to perform. However, it increases the detection of paraaortic SLNs, which is especially relevant in settings where isolated paraaortic lymph nodes are common (e.g., deep myometrial invasion or poor differentiation) (41). A systematic review by Cormier et al. (2015) demonstrated that paraaortic lymph nodes are mapped in 39\%, $2 \%$ and $17 \%$ after corporal (i.e., fundal and peritumoral), usual cervical and deeper $(3-4 \mathrm{~cm})$ cervical injection of dye (39). Considering the low risk of isolated paraaortic metastasis $(<5 \%)$ in the majority of EC patients, cervical injection of tracer is safe and accurate. Moreover, the ease of use allows for a more wide-spread implementation of the technique.

\section{Failure to map}

Mapping success depends on tumor-, patient- and surgeonspecific factors. The identification of such factors may prevent mapping failure and thereby reduce the morbidity resulting from the performance of a side-specific pelvic lymphadenectomy. Moreover, mapping of the SLN is of great importance as it is the only positive node in $46-65 \%$ of all stage IIIC cases $(42,43)$. A meta-analysis by Kang et al. (2011) demonstrated that the overall detection rate of different SLN mapping strategies (in terms of type and dose of tracer, site of injection, operation mode) is about $78 \%$. However, about $39 \%$ (range 19-80\%) fail to map bilaterally (44). Successful mapping is associated with type and site of injection of the tracer, presence of clinically enlarged lymph nodes, lymphovascular space invasion, obesity (BMI $>30 \mathrm{~kg} / \mathrm{m}^{2}$ ), history of radiation, and surgical experience of the physician. Overall, type of tracer is the most pivotal factor affecting detection rate.

Highest detection rates are achieved with a cervical injection of ICG tracer. Only paraaortic SLN detection is higher with corporal injection $(37,39,45)$.

Bilateral mapping success is lower when lymph nodes are clinically enlarged (33\%) compared to when they are not (66\%). The probability of nodal metastasis in presence of clinically enlarged lymph nodes is $25 \%$, whereas it is $4 \%$ in clinically unremarkable lymph nodes (45). Consequently, mapping failure (possibly caused by inhibited tracer flow due to lymphatic obstruction by the tumor) might correlate with the presence of metastatic disease. This has two implications. First, it is important to remove any suspicious nodes regardless of mapping (although $90 \%$ of patients with metastatic disease do not present with grossly enlarged lymph nodes). Second, the performance of a side-specific pelvic lymphadenectomy in case of bilateral mapping failure is crucial $(1,15)$.

Another reason for failed mapping is obesity. Mapping failure in obese women (BMI $>30 \mathrm{~kg} / \mathrm{m}^{2}$ ) is believed to result from an increase in adipose tissue surrounding the SLNs. This, in turn, impairs visualization of the stained SLNs. The detrimental effect of obesity on detection rate can be reduced by using ICG as a tracer $(45,46)$.

Furthermore, mapping success is lower with a history 
of radiation. Papadia et al. (2016) speculate that radiation induces fibrosis, which might alter lymphatic flow and thereby interfere with SLN mapping (28).

Last but not least, surgical experience is associated with mapping success. According to Khoury-Collado et al. (2009), there is an increase in SLN detection rate from $77 \%$ to $94 \%$ following a 30 -case experience (47).

\section{Number of SLNs to be removed and learning curve}

According to Papadia et al. (2016), the number of SLNs removed only depends on surgical experience. They suspect that with greater experience in ICG SLN mapping, the surgeon can more confidently detect and differentiate between true SLNs and more downstream echelon lymph nodes. Consequently, solely true SLNs are removed, even if more lymph nodes are stained. In fact, removing more than three SLNs per patient does not increase the accuracy of SLN mapping. This phenomenon might partly be explained due to the temporally long persistence of ICG dye and therefore staining of echelon lymph nodes, whose removal and analysis would not result in a reduction in FN rate, but adds to surgical morbidity. It follows that inexperienced surgeons cannot compensate for an inability to identify SLNs by removing additional nearby non-SLNs. As the performance of over 20 laparoscopic ICG SLN mappings resulted in retrieval of a smaller number of SLNs without increasing the $\mathrm{FN}$ rate of the procedure, Papadia et al. (2016) suggest that at least 20 SLN mapping procedures with completion lymphadenectomy should be performed before applying the SLN mapping procedure on its own. This number should be even higher in low-risk settings, as nodal metastases occur with lower frequency in these patients (48). These findings support other studies' results $(45,47)$.

\section{Pathological analysis of the SLNs and low-volume metastases}

Whenever the SLN is negative by haematoxylin-eosin stain examination, it should be further examined by ultrastaging. Ultrastaging is a more thorough pathological analysis with deeper serial sections and immunohistochemical stain for cytokeratin. Thereby, low-volume metastatic spread to SLNs can be detected. A systematic review and metaanalysis by Bodurtha et al. (2017) revealed that positive SLNs are macrometastases in 29\%, micrometastases in 39\% and isolated tumor cells in $32 \%$ of the cases (49).

Several studies examined the effect of ultrastaging on upstaging in EC. Kim et al. (2013) conducted the largest study on ultrastaging results. They found that around $40 \%$ of all patients with metastatic SLNs are detected by ultrastaging. In their cohort $12.6 \%$ of patients had metastatic disease. This translates to an upstaging of $5 \%$ of EC patients. These stage IIIC patients' low-volume metastases would have been missed by conventional pathologic processing (50). Similar numbers are described by Touhami et al. (2015). In their cohort, $44 \%$ of all patients with metastatic SLNs were detected by ultrastaging, which leads to an upstaging of $7 \%$ of patients (43). These findings are in accordance with a systematic review on the topic (39).

Due to the paucity of literature, the clinical significance and management of low-volume, ultrastage-detected metastases is not yet clear and requires long-term followup. The risk of accompanying positive non-SLNs in presence of a positive SLN depends on the size of the SLN metastasis. Overall, it is about $35-54 \%$. In presence of macrometastases it can reach up to $60.8 \%$, whereas it drops to $<5 \%$ if the SLN metastasis is $<2 \mathrm{~mm}(42,43)$.

\section{Clinical applicability and oncological outcome of SLN mapping in high- and low-risk settings}

A survey by Casarin et al. (2019) revealed that $50.3 \%$ of the surveyed gynecological oncologists apply SLN mapping as part of EC staging. Thereof, $93.1 \%$ do a cervical injection of tracer and $62.6 \%$ use ICG as a tracer. The SLN algorithm is followed by $65.0 \%$ of respondents. However, $66.7 \%$ still perform a backup lymphadenectomy in highrisk patients. Ultrastaging is part of the staging protocol in $78.9 \%$ of respondents (51). This considerable heterogenicity in terms of strategies for nodal evaluation in EC may partly be attributed to the lack of consensus on this topic in international guidelines. While some guidelines accept SLN mapping as a valid strategy for nodal assessment even in high-risk EC patients (12), others restrict its use to study settings only $(11,13)$. As a result, surgeons use a large variety of nodal evaluation strategies ranging from full bilateral pelvic and/or paraaortic lymphadenectomy, selected lymphadenectomy based on frozen section results or SLN mapping, random node sampling or no-node dissection.

There has been established evidence that SLN mapping is a safe and effective strategy for nodal evaluation in highand low-risk EC patients with appropriate sensitivity of $97.2 \%, \mathrm{FN}$ rate of $2.8 \%$ and NPV of $99.6 \%$ (9). With these numbers in mind, the risk of underdiagnosing patients due to undetected nodal metastases (i.e., FN rate of SLN 
mapping) does not exceed the risk of overtreatment and increased morbidity when performing a full systematic lymphadenectomy in every patient (i.e., risk of major complications). As EC patients have greatly different prevalences of nodal metastases, the safety of SLN mapping has been assessed for each risk category separately. Unlike high-risk EC patients, low-risk EC patients present with a low incidence of lymph node metastases. One could therefore assume that the low FN rates and the high NPVs observed in the low-risk setting could easily result from the high proportion of true negatives in this group. As a result, there was concern that the good FN rates and NPVs of SLN mapping in low-risk settings cannot be easily translated into high-risk scenarios, involving an increased number of patients with metastatic lymph nodes. The SENTI-ENDO trial (2011) contributed to this concern, as all their patients with FN results had high-risk disease (14). As a reaction, multiple institutions, including ours, evaluated the accuracy of SLN mapping in highrisk settings. We retrospectively assessed the safety of SLN mapping in a high-risk population. The sensitivity, FN rate and NPV were $90 \%, 10 \%$ and $97 \%$, respectively. The values could be lowered to $0 \%$ and raised to $100 \%$ by applying the MSKCC algorithm (i.e., removing clinically suspicious lymph nodes as well) (52). Several retro- and prospective series on SLN mapping in high-risk settings attained similar sensitivity, FN rates and NPVs, reaching from $91.2-95.8 \%, 4.2-8.8 \%$ and $96-98.6 \%$, respectively (22,53-55). In the retrospective series by Ehrisman et al. 2016, FN rate could be lowered to $0 \%$ and NPV elevated to $100 \%$ after the MSKCC algorithm was applied. This once again illustrates that, especially in high-risk settings, close adherence to the mapping algorithm is crucial. Moreover, profound knowledge of the uterine lymphatic drainage contributes to a higher accuracy of SLN mapping.

Irrespective of risk setting, the application of SLN mapping in EC staging is associated with improved oncological outcomes. A retrospective study showed that overall survival (90\% vs. 81\%), progression-free survival ( $85 \%$ vs. $75 \%)$ as well as recurrence-free survival (95\% vs. 90\%) are significantly better when SLN mapping is performed compared to full systematic lymphadenectomy alone (56). These ameliorated oncological outcomes are in accordance with other studies' findings (57-59). However, none of these studies were randomized, making them more susceptible for selection or information bias and heightening their potential for imbalance regarding prognostic factors.

\section{Conclusions}

In this review, the benefits, safety and effectiveness of SLN mapping in EC were highlighted. Not only does SLN mapping result in a reduction of surgical morbidity associated with full systematic lymphadenectomy, but it also increases the detection of metastatic disease through mapping of lymph nodes in locations not routinely removed during lymphadenectomy as well as a more thorough pathological evaluation of the SLNs. Low-risk patients benefit most from an indolent increase in detection of metastatic disease, making adjuvant treatment more tailored and increasing survival. High-risk patients benefit most from a reduction in surgical morbidity as the use of adjuvant therapy is anyway high in this setting. Moreover, the application of SLN mapping in EC staging is associated with improved oncological outcomes in terms of overall survival, progression-free survival and recurrence-free survival as compared to full systematic lymphadenectomy alone. Provided that the SLN mapping algorithm is closely adhered to, SLN mapping constitutes a safe and effective strategy for nodal evaluation in high- as well as low-risk EC, limiting both over- and undertreatment and allowing for an appropriate triage of patients to adjuvant therapy. The accuracy of SLN mapping is highest when performed through minimally invasive surgery and with a cervical injection of ICG tracer.

\section{Acknowledgments}

Funding: None.

\section{Footnote}

Provenance and Peer Review: This article was commissioned by the Guest Editors (Denis Querleu and Cherif Akladios) for the series "Sentinel Lymph Node Biopsy in Gynecologic Cancer" published in Cbinese Clinical Oncology. The article was sent for external peer review.

Conflicts of Interest: The authors have completed the ICMJE uniform disclosure form (available at http://dx.doi. org/10.21037/cco-20-224). The series "Sentinel Lymph Node Biopsy in Gynecologic Cancer" was commissioned by the editorial office without any funding or sponsorship. The authors have no other conflicts of interest to declare.

Ethical Statement: The authors are accountable for all 
aspects of the work in ensuring that questions related to the accuracy or integrity of any part of the work are appropriately investigated and resolved.

Open Access Statement: This is an Open Access article distributed in accordance with the Creative Commons Attribution-NonCommercial-NoDerivs 4.0 International License (CC BY-NC-ND 4.0), which permits the noncommercial replication and distribution of the article with the strict proviso that no changes or edits are made and the original work is properly cited (including links to both the formal publication through the relevant DOI and the license). See: https://creativecommons.org/licenses/by-nc-nd/4.0/.

\section{References}

1. Creasman WT, Morrow CP, Bundy BN, et al. Surgical pathologic spread patterns of endometrial cancer. A gynecologic oncology group study. Cancer 1987;60:2035-41.

2. FIGO stages: 1988 revision. Gynecol Oncol 1989;35:125-7.

3. Soliman PT, Frumovitz M, Spannuth W, et al. Lymphadenectomy During Endometrial Cancer Staging: Practice Patterns Among Gynecologic Oncologists. Gynecol Oncol 2010;119:291-4.

4. Ouldamer L, Bendifallah S, Body G, et al. Call for Surgical Nodal Staging in Women with ESMO/ESGO/ ESTRO High-Intermediate Risk Endometrial Cancer: A Multicenter Cohort Analysis from the FRANCOGYN Study Group. Ann Surg Oncol 2017;24:1660-6.

5. ASTEC study group, Kitchener H, Swart AM, et al. Efficacy of systematic pelvic lymphadenectomy in endometrial cancer (MRC ASTEC trial): a randomized study. Lancet 2009;373:125-36. Erratum in: Lancet 2009;373:1764.

6. Benedetti Panici P, Basile S, Maneschi F, et al. Systematic pelvic lymphadenectomy vs. no lymphadenectomy in early-stage endometrial carcinoma: randomized clinical trial. J Natl Cancer Inst 2008;100:1707-16.

7. Todo Y, Kato H, Kaneuchi M, et al. Survival effect of para-aortic lymphadenectomy in endometrial cancer (SEPAL study): a retrospective cohort analysis. Lancet 2010;375:1165-72.

8. Papadia A, Gasparri ML, Siegenthaler F, et al. FIGO stage IIIC endometrial cancer identification among patients with complex atypical hyperplasia, grade 1 and 2 endometrioid endometrial cancer: laparoscopic indocyanine green sentinel lymph node mapping versus frozen section of the uterus, why get around the problem? J Cancer Res Clin Oncol 2017;143:491-7.

9. Rossi EC, Kowalski LD, Scalici J, et al. A comparison of sentinel lymph node biopsy to lymphadenectomy for endometrial cancer staging (FIRES trial): a multicenter, prospective, cohort study. Lancet Oncol 2017;18:384-92.

10. Burke TW, Levenback C, Tornos C, et al. Intraabdominal lymphatic mapping to direct selective pelvic and paraaortic lymphadenectomy in women with high-risk endometrial cancer: results of a pilot study. Gynecol Oncol 1996;62:169-73.

11. Leitlinienprogramm Onkologie (Deutsche Krebsgesellschaft, Deutsche Krebshilfe, AWMF): Diagnostik, Therapie und Nachsorge der Patientinnen mit Endometriumkarzinom, Langversion 1.0, 2018, AWMF Registernummer: 032/034-OL.

12. Koh WJ, Abu-Rustum NR, Bean S, et al. Uterine Neoplasms, Version 1.2018, NCCN Clinical Practice Guidelines in Oncology. J Natl Compr Canc Netw 2018;16:170-99.

13. Colombo N, Creutzberg C, Amant F, et al. ESMOESGO-ESTRO Consensus Conference on Endometrial Cancer: diagnosis, treatment and follow up. Ann Oncol 2016;27:16-41.

14. Ballester M, Dubernard G, Lécuru F, et al. Detection rate and diagnostic accuracy of sentinel-node biopsy in early stage endometrial cancer: a prospective multicentre study (SENTI-ENDO). Lancet Oncol 2011;12:469-76.

15. Barlin JN, Khoury-Collado F, Kim CH, et al. The importance of applying a sentinel lymph node mapping algorithm in endometrial cancer staging: Beyond removal of blue nodes. Gynecol Oncol 2012;125:531-5.

16. Papadia A, Zapardiel I, Bussi B, et al. Sentinel lymph node mapping in patients with stage I endometrial carcinoma: a focus on bilateral mapping identification by comparing radiotracer Tc $99 \mathrm{~m}$ with blue dye versus indocyanine green fluorescent dye. J Cancer Res Clin Oncol 2017;143:475-80.

17. Eriksson AG, Beavis A, Soslow RA, et al. A Comparison of the Detection of Sentinel Lymph Nodes Using Indocyanine Green and Near-Infrared Fluorescence Imaging Versus Blue Dye During Robotic Surgery in Uterine Cancer. Int J Gynecol Cancer 2017;27:743-7.

18. Holloway RW, Ahmad S, Kendrick JE, et al. A Prospective Cohort Study Comparing Colorimetric and Fluorescent Imaging for Sentinel Lymph Node Mapping in Endometrial Cancer. Ann Surg Oncol 2017;24:1972-9.

19. Buda A, Crivellaro C, Elisei F, et al. Impact of Indocyanine Green for Sentinel Lymph Node Mapping in Early Stage 
Endometrial and Cervical Cancer: Comparison with Conventional Radiotracer (99m)Tc and/or Blue Dye. Ann Surg Oncol 2016;23:2183-91.

20. Papadia A, Imboden S, Siegenthaler F, et al. Laparoscopic Indocyanine Green Sentinel Lymph Node Mapping in Endometrial Cancer. Ann Surg Oncol 2016;23:2206-11.

21. Plante M, Touhami O, Trinh XB, et al. Sentinel node mapping with indocyanine green and endoscopic nearinfrared fluorescence imaging in endometrial cancer. A pilot study and review of the literature. Gynecol Oncol 2015;137:443-7.

22. How J, Gotlieb WH, Press JZ, et al. Comparing indocyanine green, technetium, and blue dye for sentinel lymph node mapping in endometrial cancer. Gynecol Oncol 2015;137:436-42.

23. Sinno AK, Fader AN, Roche AL, et al. A comparison of colorimetric versus fluorometric sentinel lymph node mapping during robotic surgery for endometrial cancer. Gynecol Oncol 2014;134:281-6.

24. Frumovitz M, Plante M, Lee PS, et al. Near-infrared fluorescence for detection of sentinel lymph nodes in women with cervical and uterine cancers (FILM): a randomised, phase 3, multicentre, non-inferiority trial. Lancet Oncol 2018;19:1394-403.

25. Gasparri ML, Caserta D, Panici PB, et al. Surgical staging in endometrial cancer. J Cancer Res Clin Oncol 2019;145:213-21.

26. Papadia A, Gasparri ML, Buda A, et al. Sentinel lymph node mapping in endometrial cancer: comparison of fluorescence dye with traditional radiocolloid and blue. J Cancer Res Clin Oncol 2017;143:2039-48.

27. Xiong L, Gazyakan E, Yang W, et al. Indocyanine green fluorescence-guided sentinel node biopsy: a meta-analysis on detection rate and diagnostic performance. Eur J Surg Oncol 2014;40:843-9.

28. Papadia A, Buda A, Gasparri ML, et al. The impact of different doses of indocyanine green on the sentinel lymph-node mapping in early stage endometrial cancer. J Cancer Res Clin Oncol 2018;144:2187-91.

29. Geppert B, Lönnerfors C, Bollino M, et al. A study on uterine lymphatic anatomy for standardization of pelvic sentinel lymph node detection in endometrial cancer. Gynecol Oncol 2017;145:256-61.

30. Abu-Rustum NR. Sentinel lymph node mapping for endometrial cancer: a modern approach to surgical staging. J Natl Compr Canc Netw 2014;12:288-97.

31. Cormier B, Rozenholc AT, Gotlieb W, et al; Communities of Practice (CoP) Group of Society of Gynecologic
Oncology of Canada (GOC). Sentinel lymph node procedure in endometrial cancer: A systematic review and proposal for standardization of future research. Gynecol Oncol 2015;138:478-85.

32. Rossi EC, Jackson A, Ivanova A, et al. Detection of sentinel nodes for endometrial cancer with robotic assisted fluorescence imaging: cervical versus hysteroscopic injection. Int J Gynecol Cancer 2013;23:1704-11.

33. Martinelli F, Ditto A, Bogani G, et al. Laparoscopic Sentinel Node Mapping in Endometrial Cancer After Hysteroscopic Injection of Indocyanine Green. J Minim Invasive Gynecol 2017;24:89-93.

34. How J, Boldeanu I, Lau S, et al. Unexpected locations of sentinel lymph nodes in endometrial cancer. Gynecol Oncol 2017;147:18-23.

35. Touhami O, Trinh XB, Gregoire J, et al. Predictors of non-sentinel lymph node (non-SLN) metastasis in patients with sentinel lymph node (SLN) metastasis in endometrial cancer. Gynecol Oncol 2015;138:41-5.

36. Kang S, Yoo HJ, Hwang JH, et al. Sentinel lymph node biopsy in endometrial cancer: meta-analysis of 26 studies. Gynecol Oncol 2011;123:522-7.

37. Tanner EJ, Sinno AK, Stone RL, et al. Factors associated with successful bilateral sentinel lymph node mapping in endometrial cancer. Gynecol Oncol 2015;138:542-7.

38. Eriksson AG, Montovano M, Beavis A, et al. Impact of Obesity on Sentinel Lymph Node Mapping in Patients with Newly Diagnosed Uterine Cancer Undergoing Robotic Surgery. Ann Surg Oncol 2016;23:2522-8.

39. Khoury-Collado F, Glaser GE, Zivanovic O, et al. Improving sentinel lymph node detection rates in endometrial cancer: how many cases are needed? Gynecol Oncol 2009;115:453-5.

40. Papadia A, Imboden S, Gasparri ML, et al. Endometrial and cervical cancer patients with multiple sentinel lymph nodes at laparoscopic ICG mapping: How many are enough? J Cancer Res Clin Oncol 2016;142:1831-6.

41. Walker JL, Piedmonte MR, Spirtos NM, et al. Laparoscopy compared with laparotomy for comprehensive surgical staging of uterine cancer: Gynecologic Oncology Group Study LAP2. J Clin Oncol 2009;27:5331-6.

42. Janda M, Gebski V, Davies LC, et al. Effect of Total Laparoscopic Hysterectomy vs Total Abdominal Hysterectomy on Disease-Free Survival Among Women With Stage I Endometrial Cancer: A Randomized Clinical Trial. JAMA 2017;317:1224-33.

43. Papadia A, Garbade A, Gasparri ML, et al. Minimally invasive surgery does not impair overall survival in stage 
IIIC endometrial cancer patients. Arch Gynecol Obstet 2020;301:585-90.

44. Scalici J, Laughlin BB, Finan MA, et al. The trend towards minimally invasive surgery (MIS) for endometrial cancer: an ACS-NSQIP evaluation of surgical outcomes. Gynecol Oncol 2015;136:512-5.

45. Pacini PB, Basile S, Salerno MG, et al. Secondary analyses from a randomized clinical trial: age as the key prognostic factor in endometrial carcinoma. Am J Obstet Gynecol 2014;210:363.e1-363.e10.

46. Zullo F, Falbo A, Palomba S. Safety of laparoscopy vs laparotomy in the surgical staging of endometrial cancer: a systematic review and metaanalysis of randomized controlled trials. Am J Obstet Gynecol 2012;207:94-100.

47. Soliman PT, Westin SN, Dioun S, et al. A prospective validation study of sentinel lymph node mapping for highrisk endometrial cancer. Gynecol Oncol 2017;146:234-9.

48. Gehrig PA, Cantrell LA, Shafer A, et al. What is the optimal minimally invasive surgical procedure for endometrial cancer staging in the obese and morbidly obese woman? Gynecol Oncol 2008;111:41-5.

49. Bodurtha Smith AJ, Fader AN, Tanner EJ. Sentinel lymph node assessment in endometrial cancer: a systematic review and meta-analysis. Am J Obstet Gynecol 2017;216:459476.e10.

50. Kim CH, Soslow RA, Park KJ, et al. Pathologic ultrastaging improves micrometastasis detection in sentinel lymph nodes during endometrial cancer staging. Int J Gynecol Cancer 2013;23:964-70.

51. Casarin J, Multinu F, Abu-Rustum N, et al. Factors influencing the adoption of the sentinel lymph node technique for endometrial cancer staging: an international survey of gynecologic oncologists. Int J Gynecol Cancer 2019;29:60-7.

Cite this article as: Stämpfli CAL, Papadia A, Mueller MD. From systematic lymphadenectomy to sentinel lymph node mapping: a review on transitions and current practices in endometrial cancer staging. Chin Clin Oncol 2021;10(2):22. doi: $10.21037 / \mathrm{cco}-20-224$
52. Papadia A, Gasparri ML, Radan AP, et al. Retrospective validation of the laparoscopic ICG SLN mapping in patients with grade 3 endometrial cancer. J Cancer Res Clin Oncol 2018;144:1385-93.

53. Buda A, Gasparri ML, Puppo A, et al. Lymph node evaluation in high-risk early stage endometrial cancer: A multi-institutional retrospective analysis comparing the sentinel lymph node (SLN) algorithm and SLN with selective lymphadenectomy. Gynecol Oncol 2018;150:261-6.

54. Touhami O, Grégoire J, Renaud MC, et al. Performance of sentinel lymph node (SLN) mapping in high-risk endometrial cancer. Gynecol Oncol 2017;147:549-53.

55. Ehrisman J, Secord AA, Berchuck A, et al. Performance of sentinel lymph node biopsy in high-risk endometrial cancer. Gynecol Oncol Rep 2016;17:69-71.

56. Kogan L, Matanes E, Wissing $M$, et al. The added value of sentinel node mapping in endometrial cancer. Gynecol Oncol 2020;158:84-91.

57. Buda A, Di Martino G, Restaino S, et al. The impact on survival of two different staging strategies in apparent early stage endometrial cancer comparing sentinel lymph nodes mapping algorithm and selective lymphadenectomy: An Italian retrospective analysis of two reference centers. Gynecol Oncol 2017;147:528-34.

58. Zahl Eriksson AG, Ducie J, Ali N, et al. Comparison of a sentinel lymph node and a selective lymphadenectomy algorithm in patients with endometrioid endometrial carcinoma and limited myometrial invasion. Gynecol Oncol 2016;140:394-9.

59. Imboden S, Mereu L, Siegenthaler F, et al. Oncological safety and perioperative morbidity in low-risk endometrial cancer with sentinel lymph-node dissection. Eur J Surg Oncol 2019;45:1638-43. 